\title{
ÉVALUATION ENVIRONNEMENTALE DU DEGRÉ DE CENTRALISATION D'INSTALLATIONS DE MÉTHANISATION DE DÉCHETS ORGANIQUES MÉNAGERS
}

Yves Membrez*, Michel Glauser**, Jean-Michel Imer**

Erep, Biol Conseils

L'étude est destinée à évaluer, du point de vue de l'environnement, l'influence de la centralisation des installations de méthanisation nécessaires à la digestion des déchets organiques produits par les habitants d'une région. Quatre scénarios d'organisation du traitement sont étudiés sur un canton suisse (Vaud, 600000 habitants) dans les domaines de la collecte des déchets, de leur transport, de leur méthanisation et de leur valorisation agricole. La méthode employée est celle dite des " écopoints " qui établit des " écofacteurs » tenant compte de la charge actuelle de l'environnement et de la charge critique qui le perturberait.

The purpose of the study is to evaluate, from an environmental viewpoint, the influence of the centralization of the methanization installations which are needed to eliminate the organic waste produced by the population of a given region. Four scenarios of methanization organization are studied for the canton of Vaud, Switzerland (pop. 600,000) in the domains of waste collection, transport, treatment and use for agriculture. The method used is the " ecopoint method", where " ecofactors " are established, which take into account the current impact on the environment, and the critical value of this impact that would cause environmental perturbation.

\section{INTRODUCTION}

Les déchets organiques peuvent être valorisés principalement de deux manières qui sont le compostage aérobie et la digestion anaérobie (méthanisation).

La méthanisation est notamment applicable aux déchets organiques suivants :

- fraction organique des déchets ménagers : déchets d'aliments et de préparation de repas, restes de légumes et de fruits, déchets de viande et de poisson, marc de café, sachets de thé, papier souillé ;

- déchets de cuisines en restauration collective ;

- déchets verts : gazon, herbe provenant de l'entretien des espaces verts, feuilles, déchets végétaux (écorces d'arbres, fleurs et plantes ornementales) ;
- déchets agricoles et agro-industriels : foin et paille, papier et carton, fumier, purin, fientes de volailles, marc de raisin, contenus de panses, déchets de graisses et de séparateurs, etc.

On rappellera que la digestion anaérobie est largement pratiquée dans le traitement des boues de Step et des déchets agricoles. Le biogaz produit par digestion anaérobie est généralement converti en électricité et en chaleur dans un couplage chaleur-force. D'une tonne de déchets organiques ménagers, on obtient en moyenne $100 \mathrm{~m}^{3}$ de biogaz, cette valeur pouvant osciller entre 80 et $160 \mathrm{~m}^{3}$ selon la composition des déchets.

La composition des déchets organiques introduits en digestion anaérobie peut varier fortement selon les régions (proportion des déchets de cuisines par rapport aux déchets de jardins), les types d'habitats (urbain ou rural), les systèmes de collecte proposés (composts de quartiers, « tournées vertes ", apports volontaires) et les saisons. Une étude réalisée en 1990 à Genève indique que les déchets organiques ménagers sont constitués à $30 \%$ de déchets de jardin et à

\begin{tabular}{|lr|}
\hline \multicolumn{2}{|c|}{ Tableau I } \\
\hline Désignation Composition (\%) \\
\hline Épluchures légumes & 27,1 \\
\hline Restes agrumes & 18,2 \\
\hline Déchets alimentaires & 8,8 \\
\hline Légumes + fruits entiers & 4,9 \\
\hline Pain & 3,6 \\
Os & 2,4 \\
Marc de café + sachets thé & 2,8 \\
Litières d'animaux & 2,0 \\
Plantes appartements + fleurs & 7,2 \\
\hline Terre (plantes appartement) & 6,1 \\
Non triables & 12,8 \\
Sacs poubelles & 1,3 \\
Sacs plastiques & 0,1 \\
Emballages carton & 0,3 \\
Papier & 2,2 \\
Indésirables, verre, etc. & 0,2 \\
\hline Total & 100 \\
\hline
\end{tabular}

$70 \%$ de déchets de cuisine (\% du poids total), et que la composition typique des déchets de cuisine est celle du tableau I.

La teneur en eau de ce mélange est de $69,1 \%$, alors que sa teneur en matière organique se situe à $73,8 \%$ de la matière sèche, et que son rapport $\mathrm{C} / \mathrm{N}$ (carbone) azote) est de 18,0.

Outre le biogaz, la digestion anaérobie produit un compost. Après une période de maturation aérobie de deux à trois semaines, la structure du produit ainsi que les sub- 


\begin{tabular}{|lcc|}
\hline \multicolumn{3}{|c|}{ Tableau 2} \\
\hline & $\begin{array}{c}\text { Compost } \\
\text { aérobie } \\
\text { (53 analyses) }\end{array}$ & $\begin{array}{c}\text { Compost } \\
\text { anaérobie } \\
\text { (29 analyses) }\end{array}$ \\
\hline Matière sèche (\% matière fraîche) & 51,00 & 50,50 \\
\hline Matière organique (\% matière sèche) & 46,00 & 51,40 \\
\hline Azote total (kg/t MS) & 13,00 & 12,00 \\
\hline Nitrate $\mathrm{NO}_{3}-\mathrm{N}$ (g/t MS) & 49,70 & 9,20 \\
\hline Ammonium NH$/ \mathrm{N}$ (g/t MS) & 148,00 & 707,00 \\
\hline Azote minéral (g/t MS) & 198,00 & 716,00 \\
\hline Phosphore (kg/t MS) & 6,20 & 7,40 \\
\hline Potassium, en K2O (kg/t MS) & 10,80 & 9,15 \\
\hline Calcium (kg/t MS) & 53,00 & 50,70 \\
\hline Magnésium $(\mathrm{kg} / \mathrm{t} \mathrm{MS})$ & 7,60 & 6,90 \\
\hline
\end{tabular}

stances qu'il contient son comparables à celles d'un compost aérobie mûri pendant six mois. Les résultats des analyses effectuées en 1996 sur les installations de compostage et de méthanisation du canton de Zurich illustrent ces dires (tableau 2).

L'objectif du présent projet est d'inclure les aspects environnementaux dans les critères d'évaluation à prendre en compte lors de l'établissement d'un concept directeur de traitement des déchets organiques ménagers par méthanisation. L'étude tente de répondre à la question suivante : pour une région donnée, compte tenu de sa population et de sa structure d'habitat, est-il préférable, du point de vue de l'environnement, de traiter par méthanisation les déchets organiques dans des installations décentralisées ou, au contraire, dans des installations centralisées de grande capacité ?

\section{MÉTHODES}

Les écobilans sont calculés avec la méthode dite des écopoints de l'OFEFPa (1991). Celle-ci permet de pondérer les diverses charges environnementales liées à une activité et de les agréger de manière à obtenir une valeur unique représentant un indice de performance environnementale globale.

Les facteurs de pondération, appelés écofacteurs, prennent en compte la capacité de l'environnement à supporter une émission donnée (flux critique) et le niveau actuel de l'émission (flux actuel). L'écofacteur EF peut être exprimé par l'équation suivante :

$\mathrm{EF}=\mathrm{k} \cdot \mathrm{Ca} /(\mathrm{Cc})^{2}$

où

$\mathrm{k}$ : facteur de normalisation sans unité (facteur identique pour toutes les émissions);

Ca : charge actuelle :

$\mathrm{Cc}_{\mathrm{C}}$ : charge critique encore acceptable par l'environnement.

Dans la méthode de l'OFEFP, les charges sont définies pour le territoire suisse. Un écofacteur peut être défini pour tout impact sur l'environnement naturel, donc pour chacune des émissions de polluants dans l'air, l'eau, le sol, pour la pro- duction de déchets solides, la consommation d'énergie et l'utilisation du sol et des matières premières. L'indice de charge environnementale est obtenu en multipliant une émission ou une consommation par l'écofacteur correspondant. Ce résultat est exprimé en écopoints (EP). Les charges environnementales prises en compte correspondent pour une grande part à celles proposées par l'OFEFPa (I99|).

En fonction de l'offre actuelle du marché, les installations de digestion ont été classées en 4 catégories selon leur capacité de traitement :

- installations supra-régionales d'une capacité de traitement de 20000 à 50000 t de matière fraîche par an (MF/an);

- installations régionales d'une capacité de traitement de 10000 à 20000 t MF/an ;

- installations communales d'une capacité de traitement de 2000 à 5000 t MF/an ;

- installations agricoles d'une capacité de traitement de 50 à $200 \mathrm{t}$ MF/an.

Sur la base de cette classification, 4 scénarios ont été établis pour méthaniser les 52000 t/an de déchets organiques ménagers des 600000 habitants du canton de Vaud.

Chaque scénario ne comprend que des installations d'une même capacité de traitement : le scénario I des installations supra-régionales, le scénario 2 des installations régionales, le scénario 3 des installations communales et le 4 des installations agricoles (figure I).

Les installations des scénarios I, 2 et 3 sont de type industriel (degré d'automatisation élevé, alimentation en continu). La description des scénarios est faite par calcul pour la collecte et les transports des déchets et des résidus. Les caractéristiques des différentes installations (construction et exploitation) sont obtenues sur la base d'un questionnaire rempli par 17 constructeurs européens.

\section{RÉSULTATS ET DISCUSSION}

Les émissions dans l'air et les eaux produites lors des phases de collecte, de transport et de traitements de déchets organiques ménagers, ainsi que celles produites pour l'évacuation des résidus à valoriser figurent dans les tableaux 3 et 4 (ainsi que les écofacteurs retenus pour chaque polluant).

Les valeurs négatives signifient que les émissions sont inférieures à ce qu'elles seraient si l'électricité et la chaleur produites par la digestion des déchets organiques provenaient respectivement du réseau et de chaudières à mazout.

Pour le $\mathrm{SO}_{2}$, les $\mathrm{NMCOV}$ et les $\mathrm{CO}_{x}$, le scénario 4 est le plus

\begin{tabular}{|c|c|c|c|c|c|}
\hline & \multicolumn{2}{|c|}{ Scénario Scénaric } & \multirow{2}{*}{$\begin{array}{c}\text { Scénario } \\
3\end{array}$} & \multicolumn{2}{|c|}{ Scénario Écofacteurs } \\
\hline & I & 2 & & 4 & \\
\hline $\mathrm{SO}_{2}[\mathrm{~kg} / \mathrm{an}]$ & -465 & -788 & -1000 & -7900 & 23 \\
\hline $\mathrm{NO}_{x}[\mathrm{~kg} / \mathrm{an}]$ & 34400 & 31800 & 29900 & 89300 & 42,3 \\
\hline NMCOV*[kg/ & /an] -2900 & -3700 & -4200 & -8300 & 14,3 \\
\hline $\mathrm{CH}_{4}[\mathrm{~kg} / \mathrm{an}]$ & 24400 & 24400 & 24400 & 26500 & 2,1 \\
\hline $\mathrm{CO}_{x}[\mathrm{~kg} / \mathrm{an}]$ & -3500000 & -3700000 & -3900000 & -5000000 & 0,036 \\
\hline
\end{tabular}


Scénario I : I installation supra-régionale dans le district de Morges

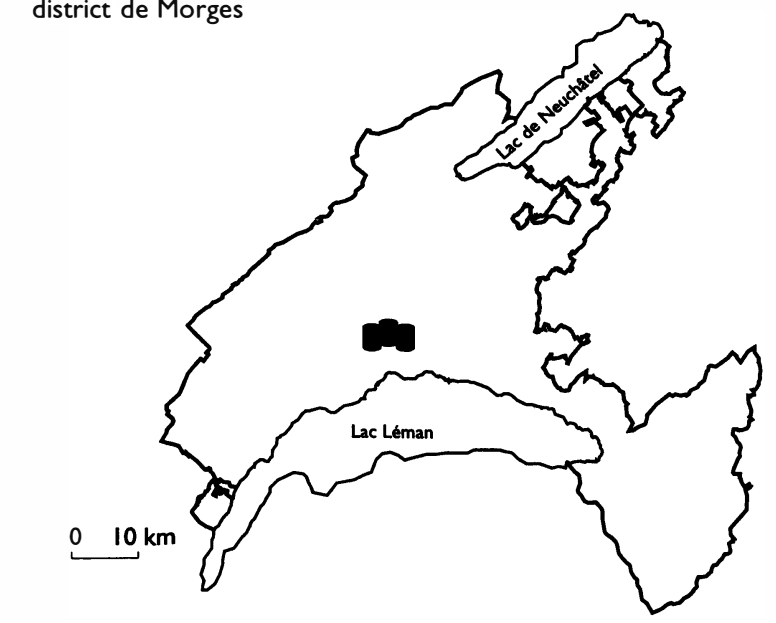

Scénario 3 : 12 installations communales

(capacité comprise entre 2000 et 5000 t/an)

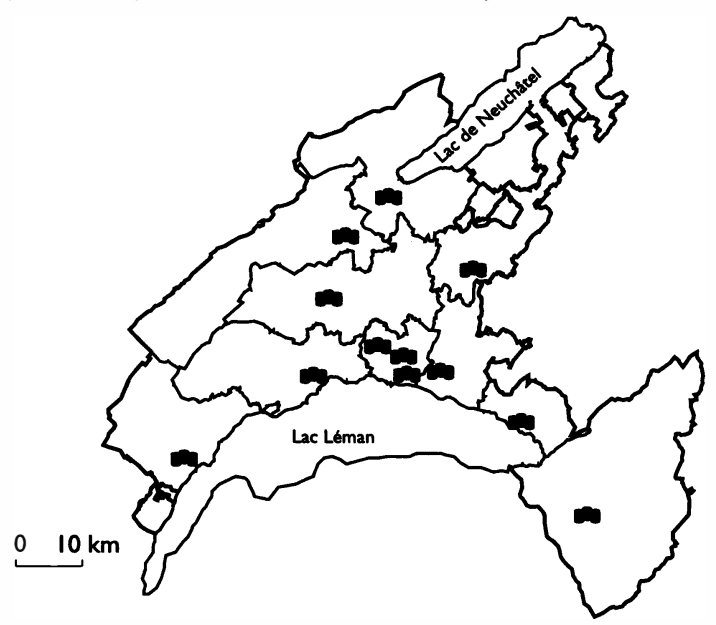

Figure I : Quatre scénarios de méthanisation

favorable (moins de transports). Cela n'est cependant pas le cas pour les émissions de $\mathrm{NO}_{x}$ et de $\mathrm{CH}_{4}$. Ceci provient du fait que les couplages chaleur-force de faible puissance ne sont pas aussi performants que ceux de puissance élevée. Ces derniers doivent en effet respecter les exigences de l'Ordonnance sur la protection de l'air (1985) à partir d'une consommation de carburant de plus de $10 \mathrm{~kg} / \mathrm{h}$ pour les moteurs à allumage commandé et de $50 \mathrm{~kg} / \mathrm{h}$ pour les moteurs à allumage spontané.

Pour les scénarios I, 2 et 3 , on a admis que la production d'eau en excès est de $0,4 \mathrm{~m}^{3}$ par tonne de déchets organiques traités. Pour le scénario 4 , on a admis que les eaux en excès sont utilisées comme fumure dans les exploitations agricoles. Dans ce dernier cas, la charge directe sur les eaux est donc nulle (si l'épandage est pratiqué dans de bonnes conditions, ce qui est admis ici).

Les émissions de substances dans les eaux figurent dans le tableau 4.

Les bonus (valeurs négatives) indiqués pour le scénario 4 pro-
Scénario $2: 4$ installations régionales dans les districts de - Lausanne

- Morges

- Vevey

- Orbe

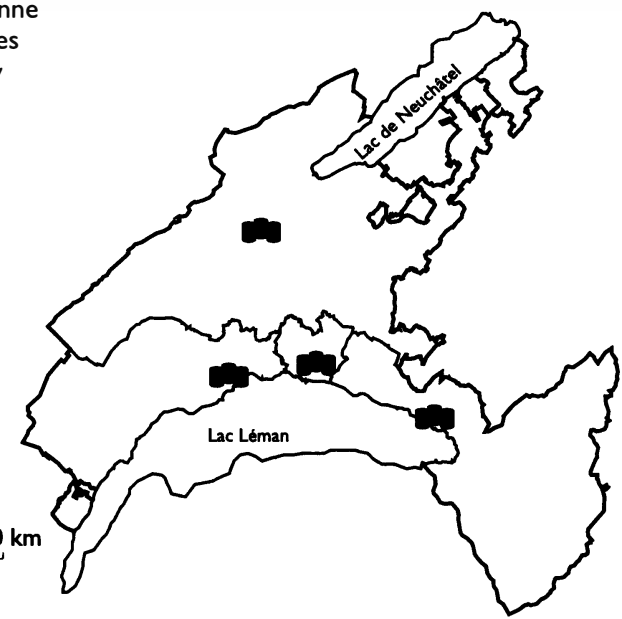

Scénario $4: 500$ installations agricoles, traitant des déchets organiques ménagers en plus des déchets de ferme, réparties sur l'ensemble du canton

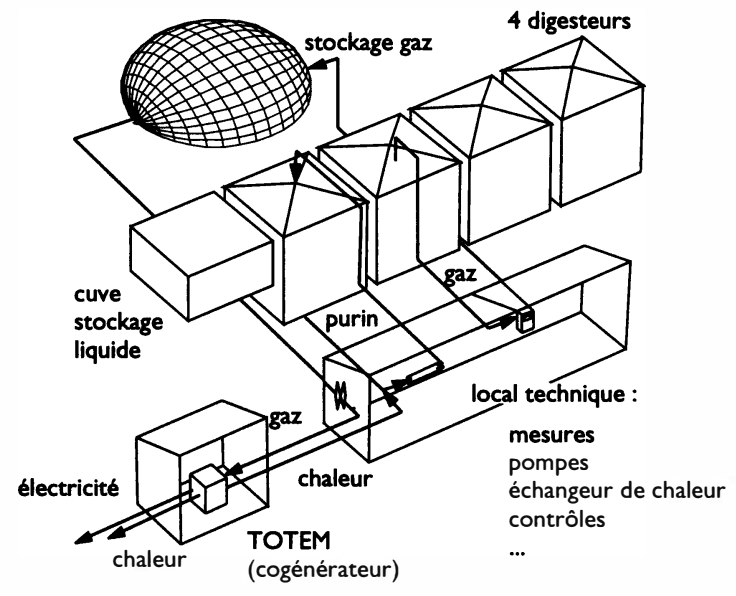

\begin{tabular}{|c|c|c|c|}
\hline & Scénarios 1,2 et 3 & Scénario 4 & Écofacteurs \\
\hline $\mathrm{DOC}$ [ $\mathrm{kg} \mathrm{C} / \mathrm{an}]$ & 4100 & -120 & 11,5 \\
\hline $\mathrm{NH}_{4}{ }^{+}[\mathrm{kg} \mathrm{N} / \mathrm{an}]$ & 16700 & -35 & 10,3 \\
\hline Sulfates [kg/an] & 8100 & .290 & 0,077 \\
\hline Nitrates [kg/an] & 930 & -13 & 0,91 \\
\hline Chlorures [kg/an] & 4700 & -570 & 0,026 \\
\hline Phosphore [kg/an] & 210 & -160 & 756 \\
\hline
\end{tabular}

viennent de la précombustion des agents énergétiques. En effet, les économies d'énergie réalisées avec le biogaz étant comptabilisées dans l'écobilan, les émissions dans les eaux qui leur sont liées pour la précombustion des agents énergétiques (extraction raffinage, transport) sont négatives. Comme on a admis qu'il n'y avait aucun rejet d'eaux de processus pour le scénario 4 , le résultat final reste donc négatif.

Le fait que les ressources énergétiques ne soient pas infinies justifie que la rareté de l'énergie soit prise en compte dans 
l'écobilan (limites des gisements d'énergies fossiles, limites des possibilités de réalisation de nouvelles centrales hydroélectriques). Les nuisances découlant de la consommation d'énergie sont enregistrées aux comptes de la pollution de l'air et de la pollution des eaux.

Le critère de rareté de l'énergie est basé sur la consommation d'énergie primaire. Pour établir cette valeur sur la base des consommations d'énergies finales, on a tenu compte des rendements de la conversion des énergies primaires (force hydraulique, minerai d'uranium, pétrole brut) en énergies finales (électricité, mazout).

Les quantités d'énergie primaire ont été calculées en admettant un rendement moyen de $90 \%$ pour la production de diesel et de mazout et de $38 \%$ pour l'électricité (OFEFPb, 1991). Les résultats pour chaque scénario figurent dans le tableau 5.

\begin{tabular}{|c|c|c|c|c|}
\hline $\begin{array}{c}\text { Scénario I } \\
\mid\end{array}$ & $\begin{array}{c}\text { Scénario } 2 \\
2\end{array}$ & $\begin{array}{c}\text { Scénario } \\
3\end{array}$ & $\begin{array}{c}\text { Scénario } \\
4\end{array}$ & $\begin{array}{l}\text { Éco- } \\
\text { facteurs }\end{array}$ \\
\hline \multicolumn{5}{|c|}{ Consommation d'énergie primaire [kWh/an] } \\
\hline-18100000 & -19000000 & -19600000 & -26500000 & 3,6 \\
\hline
\end{tabular}

On constate que le scénario 4 permet les plus grandes économies d'énergie primaire.

La charge environnementale due au bruit routier est exprimée en terme de prestations de circulation ( $\mathrm{km}$ parcourus).

\begin{tabular}{|c|c|c|c|c|}
\hline Scénario I & $\begin{array}{c}\text { Scénario } 2 \\
2\end{array}$ & $\begin{array}{c}\text { Scénario } \\
3\end{array}$ & $\begin{array}{c}\text { Scénario } \\
4\end{array}$ & $\begin{array}{c}\text { Éco- } \\
\text { facteurs }\end{array}$ \\
\hline \multicolumn{5}{|c|}{ Prestations de circulation des camions [km/an] } \\
\hline 837000 & 573000 & 389000 & 312000 & 120 \\
\hline
\end{tabular}

Les trajets effectués pour la collecte ont été calculés en multipliant les temps de collecte par une vitesse moyenne de $6 \mathrm{~km} / \mathrm{h}$. Le rapport des prestations de circulation du scénario I sur celles du scénario 4 est de 2,7.

Les charges environnementales présentées plus haut ont été pondérées avec les écofacteurs correspondants et additionnées pour obtenir un indice de charge environnementale globale. On notera qu'un résultat élevé exprimé en écopoints correspond à une atteinte environnementale élevée. Les résultats sont présentés tout d'abord pour les domaines environnementaux Air (tableau 7) et Eaux (tableau 8). On peut constater que les émissions de $\mathrm{NO}_{x}$ prédominent

\begin{tabular}{|c|c|c|c|c|}
\hline \multicolumn{5}{|c|}{$\begin{array}{l}\text { Tableau } 7 \text { : Charges environnementales } \\
\text { des émissions atmosphériques }\end{array}$} \\
\hline & $\begin{array}{c}\text { Scénario } \\
\text { | }\end{array}$ & $\begin{array}{c}\text { Scénario } \\
2\end{array}$ & $\begin{array}{c}\text { Scénario } \\
3\end{array}$ & $\begin{array}{c}\text { Scénario } \\
4\end{array}$ \\
\hline $\mathrm{SO}_{2}$ [Mio EP] & -11 & -18 & 23 & -181 \\
\hline NO [Mio EP] & 1454 & 1344 & 1266 & 3776 \\
\hline NMCOV [Mio EP] & -42 & -53 & -61 & -118 \\
\hline $\mathrm{CH}_{4}$ [Mio EP] & 51 & 51 & 51 & 56 \\
\hline $\mathrm{CO}_{x}$ [Mio EP] & -126 & -134 & -140 & -179 \\
\hline Total [Mio EP] & 1327 & 1190 & 1094 & 3352 \\
\hline
\end{tabular}

\begin{tabular}{|c|c|c|}
\hline \multicolumn{3}{|c|}{$\begin{array}{c}\text { Tableau } 8 \text { : Charges environnementales } \\
\text { des émissions dans les eaux }\end{array}$} \\
\hline & Scénarios I, 2 et 3 & Scénario 4 \\
\hline DOC [Mio EP] & 47 & $-1,3$ \\
\hline $\mathrm{NH}_{4}^{+}$[Mio EP] & 172 & $-0,4$ \\
\hline Sulfates [Mio EP] & 0,6 & $-0,02$ \\
\hline Nitrates Mio EP] & 0,8 & $-0,01$ \\
\hline Chlorures [Mio EP] & 0,1 & $-0,01$ \\
\hline Phosphore [Mio EP] & 158 & $-0,1$ \\
\hline Total [Mio EP] & 379 & $-1,8$ \\
\hline
\end{tabular}

dans le bilan total. Le scénario 4 est largement le moins favorable. Comme on l'a vu plus haut, ceci provient du coefficient d'émission appliqué aux couplages chaleur-force de faible puissance.

Pour les scénarios I, 2 et 3, les émissions de $\mathrm{NH}_{4}{ }^{+}$et de phosphore prédominent largement. Le scénario 4 est le plus favorable. Ceci provient du fait qu'il ne produit pas d'eau de processus en excès à évacuer dans une canalisation.

Les charges environnementales induites par la consommation d'énergie primaire et par le bruit routier s'obtiennent directement en multipliant les valeurs des tableaux 5 et 6 par les écofacteurs correspondants.

Le tableau 9 présente la synthèse des résultats.

\begin{tabular}{|lcccc|}
\hline \multicolumn{5}{c|}{ Tableau 9 : Ecobilan présenté par domaine } \\
environnemental \\
\hline & Scénario & Scénario & Scénario & Scénario \\
& 1 & $\mathbf{2}$ & $\mathbf{3}$ & $\mathbf{4}$ \\
Air [Mio EP] & 1327 & 1190 & 1094 & 3352 \\
Eau [Mio EP] & 379 & 379 & 379 & $-1,9$ \\
Énergie [Mio EP] & -65 & -68 & -70 & -96 \\
Bruit [Mio EP] & 100 & 69 & 47 & 37 \\
Total [Mio EP] & 1741 & 1570 & 1449 & $\mathbf{3 2 9 2}$ \\
\hline
\end{tabular}

Sur la base de ces résultats, on peut faire les commentaires suivants :

- les charges sur l'air sont prédominantes ;

- les bonus issus de la production d'une forme d'énergie renouvelable compensent en moyenne la charge environnementale due au bruit du trafic routier ;

- le scénario 3 est le plus avantageux, sa charge environnementale est inférieure de $8 \%$ à celle du scénario 2 qui le précède ;

- le scénario 4 est largement le plus désavantageux, sa charge est $88 \%$ plus élevée que celle du scénario I qui le précède, soit presque le double.

\section{CONCLUSIONS}

I. La charge environnementale du traitement des déchets organiques ménagers par méthanisation est dominée par le problème de la qualité de l'air. En conséquence, deux paramètres sont déterminants pour l'évaluation des scénarios: a) l'importance de la collecte des déchets et de leur transport ;

b) la qualité des émissions atmosphériques des couplages cha- 
leur-force (CCF).

2. En conséquence, l'écobilan du scénario le plus centralisateur ( I) est chargé par les transports entre les producteurs de déchets et le centre de traitement, ainsi que par ceux du centre de traitement jusqu'aux lieux de valorisation des composts.

3. En conséquence encore, l'écobilan du scénario le plus décentralisateur (4), où les déchets sont traités dans de petites installations, est chargé par les émissions atmosphériques de $\mathrm{NO}_{x}$ des CCF. En effet, la législation suisse n'oblige pas les petits CCF à être équipés de catalyseurs, alors que c'est le cas pour les gros. Les émissions des petits peuvent alors être 100 fois plus importantes que celles des gros.

4. Pour le canton de Vaud, on constate que le meilleur niveau de centralisation serait celui du scénario 3 , soit une douzaine d'installations traitant entre 2000 et 5000 t/an. Ce scénario pourrait être mixé avec les 2 et 4 en remplaçant quelques installations communales par une ou deux installations régionales dans des régions urbanisées et en ajoutant des petites installations agricoles en campagne. Ces dernières devraient alors être équipées de catalyseurs pour que l'écobilan reste favorable.

5. La méthode des écopoints est adaptée au problème posé pour la Suisse et a permis de conclure pour le canton de Vaud. En ajustant les paramètres de calcul, elle pourra être adaptée pour d'autres régions.

Pour être généralisés à d'autres régions du monde, les écofacteurs devraient être révisés pour tenir compte des changements d'échelle et de l'importance relative des problèmes environnementaux.

\section{* Yves Membrez}

Erep SA - Chemin du Coteau 28 - II23 Aclens - Suisse

\section{** Michel Glauser}

Biol conseils SA - Rue du Lac 6 - 1400 Yverdon-les-Bains - Suisse

\section{** Jean-Michel Imer}

Biol conseils SA - Rue de la Serre 5 - 2000 Neuchâtel - Suisse

Remerciements

Ce projet a pu être réalisé grâce au soutien de l'Office fédéral de l'énergie - Section énergies renouvelables.

\section{Bibliographie}

- OFEFPa, (1991), Office fédéral de l'environnement, des forêts et du paysage, Méthodologie des écobilans sur la base de l'optimisation écologique, Cahier de l'environnement, 133.

- OFEFPb, (199|), Office fédéral de l'environnement, des forêts et du paysage, Déchets, Bilan écologique des matériaux d'emballage état en 1990, Cahier de l'environnement, 132.

- OPair, (1985), Ordonnance fédérale sur la protection de l'air ( 16 décembre).

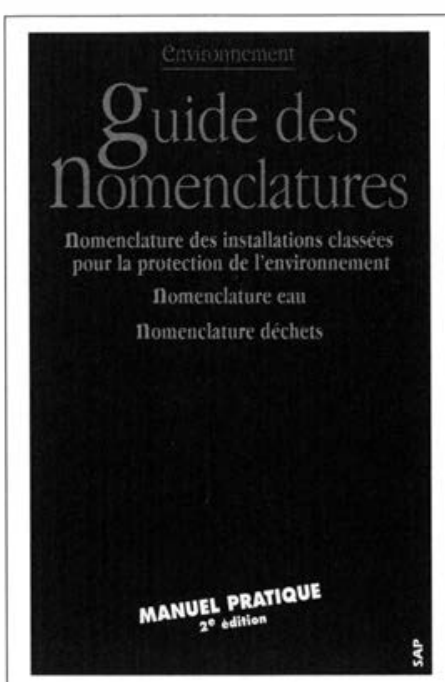

Les nomenclatures installations classées et les nomenclatures eau et déchets sont présentées dans un seul ouvrage mis à jour de toutes modifications réglementaires intervenues en 1997. Présentées sous une forme didactique et pratique l'édition 1998 de cet ouvrage unique est d'un grand secours pour les milliers d'utilisateurs, potentiels prescripteurs, industriels, collectivités, organismes consulaires, administrations, etc., qui font face à la difficulté de saisir et d'utiliser efficacement, sans oubli et sans erreur toutes les subtilités et exigences réglementaires imposées pour la protection de l'environnement. Les auteurs Gabriel Ullmann de EEC Consultants et Thierry Murat du Cabinet Murat ont assuré la mise à jour de cette $\mathbf{2}^{\mathrm{e}}$ édition

- Installations classées : tableau de conversion nouvelles/anciennes rubriques et nomenclature des ICPE

- Eau : nomenclature avec classement numérique des rubriques et classement alphabétique

- Déchets : nomenclature (comprenant DIS) avec classement numérique des rubriques

Format $15^{\star} 24$ - 125 pages - 178 F TTC franco de port (168,72 F HT - TVA 5,5 \% : 9,28 F)

\section{SAP - 7, chemin de Gordes - 38100 Grenoble Tél. 0476432864 - Fax 0476569409}

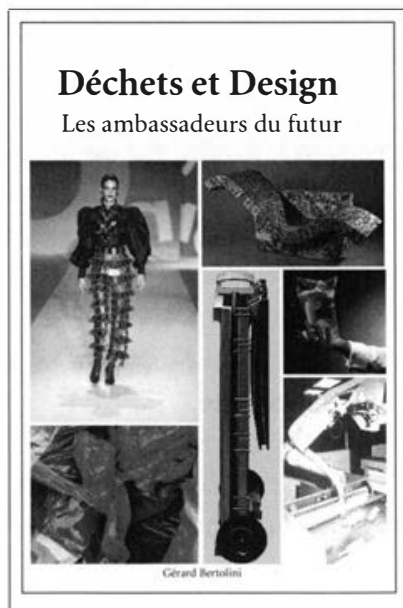

Rapprocher déchet et design peut paraître singulier. Pourtant, le déchet intervient comme mécanisme de rappel; au-delà, il interpelle les designers, et plus largement les créateurs. De plus en plus, ils devront avoir une démarche prospective, anticipatrice, préventive, remettre en cause la conception des produits pour mieux tenir compte de l'après-usage et des préoccupations environnementales, qui constituent des valeurs d'avenir. Et puis, une fois n'est pas assez. Réemploi, réutilisation, recyclage, boucles et cascades, les produits doivent devenir des re-produits, les créateurs des re-créateurs.

Enfin, le produit doit être efficace dans sa mission, ainsi que dans sa démission. Pour les déchets ultimes (à réduire autant que possible), leur concours peut là encore être précieux.

Pour libérer l'avenir, le design doit investir le champ du déchet nous dit Gérard Bertolini, économiste, directeur de recherche au CNRS et spéculateur sur l'avenir des déchets.

Format $15,5^{\star} 24-204$ pages - $179 \mathrm{~F}$ TTC franco de port $(169,67 \mathrm{~F} \mathrm{HT}-$ TVA $5,5 \%: 9,33 \mathrm{~F})$

SAP - 7, chemin de Gordes - 38100 Grenoble Tél. 0476432864 - Fax 0476569409 